Pacific Journal of Mathematics

SOME IDENTITIES VALID IN SPECIAL JORDAN ALGEBRAS
BUT NOT VALID IN ALL JORDAN ALGEBRAS 


\title{
SOME IDENTITIES VALID IN SPECIAL JORDAN ALGEBRAS BUT NOT VALID IN ALL JORDAN ALGEBRAS
}

\author{
C. M. Glennie
}

A Jordan algebra is defined by the identities:

$$
x \cdot y=y \cdot x,(x \cdot y) \cdot y^{2}=\left(x \cdot y^{2}\right) \cdot y .
$$

The algebra $A_{J}$ obtained from an associative algebra $A$ on replacing the product $x y$ by $x \cdot y=1 / 2(x y+y x)$ is easily seen to be a Jordan algebra. Any subalgebra of a Jordan algebra of this type is called special. It is known from work of Albert and Paige that the kernel of the natural homomorphism from the free Jordan algebra on three generators to the free special Jordan algebra on three generators is nonzero and consequently that there exist three-variable relations which hold identically in any homomorphic image of a special Jordan algebra but which are not consequences of the defining identities (1). Such a relation we shall call an $S$-identity. It is the purpose of this paper to establish that the minimum possible degree for an $S$-identity is 8 and to give an example of an $S$-identity of degree 8 . In the final section we use an $S$-identity to give a short proof of the main theorem of Albert and Paige in a slightly strengthened form.

Notation. The product in a Jordan algebra will be denoted by a dot, thus $a \cdot b$, and $\{a b c\}$ will denote the Jordan triple product

$$
\{a b c\}=a \cdot(b \cdot c)-b \cdot(c \cdot a)+c \cdot(a \cdot b) .
$$

Unbracketed products $a_{1} \bullet a_{2} \cdot \cdots \cdot a_{n}$ will denote left-normed products i.e. $\left(\cdots\left(\left(a_{1} \cdot a_{2} \cdot\right) \cdot a_{3}\right) \cdot \cdots \cdot a_{n}\right)$. When working in a special Jordan algebra we shall use juxtaposition, thus $a b$, to denote the product in the underlying associative algebra. Then $a \cdot b=1 / 2(a b+b a)$ and $2\{a b c\}=$ $a b c+c b a$. The free (respectively free special) Jordan algebra on $n$ generators, taken as $x_{1}, \cdots, x_{n}$ or as $x, y, z$ if $n=3$, will be denoted by $J^{(n)}$ (respectively $J_{0}^{(n)}$ ) and the kernel of the natural homomorphism $\nu_{n}$ (written as $\nu$ for $n=3$ ) of $J^{(n)}$ onto $J_{0}^{(n)}$ by $K_{n}$. The subspace of $J^{(n)}$ spanned by the monomials of degree $n$ linear in each of the generators will be denoted by $L_{n}$. The underlying associative algebra for $J_{0}^{(n)}$ is the free associative algebra on $n$ generators: we shall denote this by $A^{(n)}$. Throughout the paper we work over some fixed, but arbitrary, field of characteristic not two.

Received August 13, 1964. This paper is a revised version of part of the author's 1963 Yale Ph.D. dissertation. 
1. The following theorem has been proved by MacDonald [4]:

Theorem 1. (MacDonald). $K_{3}$ contains no (nonzero) element which is linear in one of the generators.

We have at once the following corollaries:

Corollary 1. $K_{3}$ contains no (nonzero) element of degree less than six.

CoRollary 2. An element $u$ in $J^{(3)}$ linear in one generator, or of degree less than six, can be unambiguously represented by the expansion of us in $A^{(3)}$.

In this section we shall strengthen Corollary 1 to the following theorem, which I understand has previously been proved by J. Blattner:

Theorem 2. $K_{3}$ contains no (nonzero) element of degree less than eight.

Proof. Let $L$ be the subspace of $J^{(3)}$ spanned by the elements of degree two in $x$, two in $y$ and two in $z ; M$ the subspace of $J^{(3)}$ spanned by the elements of degree two in $x$, two in $y$ and three in $z$. It is sufficient to show that (i) the restriction of $\nu$ to $L$ is one-to-one and (ii) the restriction of $\nu$ to $M$ is one-to-one. For (i) we display a set of elements which span $L$ but whose images are linearly independent in $L \nu$. For (ii) we prove a Lemma which implies that if (ii) does not hold, then (i) does not hold.

Let $R_{b}$ denote the mapping $a \rightarrow a \cdot b$ in a Jordan algebra. Then it is well-known that:

$$
\begin{aligned}
& R_{a \cdot b \cdot c}=R_{a \cdot b} R_{c}+R_{b \cdot c} R_{a}+R_{c \cdot a} R_{b}-R_{a} R_{c} R_{b}-R_{b} R_{c} R_{a} \\
& R_{a \cdot b \cdot c}=R_{a} R_{b \cdot c}+R_{b} R_{c \cdot a}+R_{c} R_{a \cdot b}-R_{a} R_{c} R_{b}-R_{b} R_{c} R_{a} \\
& R_{a \cdot b} R_{c}+R_{b \cdot c} R_{a}+R_{c \cdot a} R_{b}=R_{a} R_{b \cdot c}+R_{b} R_{c \cdot a}+R_{c} R_{a \cdot b}
\end{aligned}
$$

So $L$ is spanned by elements of the forms
(i) $a R_{b} R_{c} R_{d} R_{e} R_{b}$
( v ) $a R_{b} R_{c} R_{d} R_{e \cdot f}$
(ii) $a R_{b . c} R_{d} R_{e} R_{f}$
(vi) $a R_{b \cdot c} R_{d \cdot e} R_{f}$
(iii) $a R_{b} R_{c \cdot d} R_{e} R_{f}$
(vii) $a R_{b \cdot c} R_{d} R_{e \cdot f}$
(iv) $a R_{b} R_{c} R_{d \cdot e} R_{f}$
(viii) $\quad a R_{b} R_{c \cdot d} R_{e \cdot f}$ 
where two of $a, b, c, d, e, f$ represent $x$, two represent $y$ and two represent $z$. Consider those of type (i). We have

$$
\begin{aligned}
\left(16 a R_{b} R_{c} R_{d} R_{e}\right) \nu & =(a b c d e+e d c b a)+(b a c d e+e d c a b) \\
& +(c a b d e+e d b a c)+(c b a d e+e d a b c) \\
& +(d a b c e+e c b a d)+(d b a c e+e c a b d) \\
& +(d c a b e+e b a c d)+(d c b a e+e a b c d) \\
& =U_{1}+U_{2}+\cdots+U_{8} \text { (say) } \\
\text { (where } & \left.U_{1}=a b c d e+e d c b a, \text { etc }\right) .
\end{aligned}
$$

Cohn has shown [2] that reversible elements in $A^{(3)}$ are in $J_{0}^{(3)}$ so that each $U_{i}$ is in $J_{0}^{(3)}$. Since $\nu$ is an epimorphism there exist $u_{i} \in J^{(3)}(i=$ $1, \cdots, 8)$ for which $u_{i} \nu=U_{i}$. Then

$$
\left(16 a R_{b} R_{c} R_{d} R_{e}\right) \nu=\Sigma U_{i}=\Sigma\left(u_{i} \nu\right)=\left(\Sigma u_{i}\right) \nu
$$

Thus

$$
16 a R_{b} R_{c} R_{d} R_{e}=\Sigma u_{i} \quad \text { (Theorem 1, Corollary 1) }
$$

and

$$
16 a R_{b} R_{c} R_{d} R_{e} R_{f}=\left(\Sigma u_{i}\right) \cdot f=\Sigma\left(u_{i} \cdot f\right)
$$

By Theorem 1, Corollary 2, we can use $U_{i}$ to represent $u_{i}$ without introducing ambiguity. Thus instead of $u_{1} \cdot f$ we can write $U_{1} \cdot f$ i.e. $(a b c d e+e d c b a) \cdot f$, an element in $J^{(3)}$ but with notation for the part in brackets borrowed from $J_{0}^{(3)}$. Treating elements of types (ii)-(viii) similarly we see that with this notational convention $L$ is spanned by elements of the forms $(a b c d e+e d c b a) \cdot f$ and $(a b c d+d c b a) \cdot(e \cdot f)$. The following elements then, together with those obtained by permuting $x, y$ and $z$, span $L$ :

\section{T-elements}

1. $2 x \cdot\left(x y^{2} z^{2}+z^{2} y^{2} x\right)=4 x \cdot\left\{x y^{2} z^{2}\right\}$

2(a). $\quad x \cdot(x y z y z+z y z y x)=2 x \cdot\{x\{y z y\} z\}=2 x \cdot\{x y\{z y z\}\}$.

(b). $x \cdot(x z y z y+y z y z x)=2 x \cdot\{x\{z y z\} y\}=2 x \cdot\{x z\{y z y\}\}$

3. $\quad 2 x \cdot\left(x y z^{2} y+y z^{2} y x\right)=4 x \cdot\left(x \cdot\left\{y z^{2} y\right\}\right)$

4. $\quad 2 z \cdot\left(y z y x^{2}+x^{2} y z y\right)=4 z \cdot\left(\{y z y\} \cdot x^{2}\right)$

5. $2 x \cdot(y x z y z+z y z x y)=4 x \cdot\{y x\{z y z\}\}$

6. $2 y \cdot\left(z y x^{2} z+z x^{2} y z\right)=4 y \cdot\left\{z\left(y \cdot x^{2}\right) z\right\}$

7. $2 x \cdot\left(y^{2} x z^{2}+z^{2} x y^{2}\right)=4 x \cdot\left\{y^{2} x z^{2}\right\}$

8(a). $\quad x \cdot(y z x y z+z y x z y)=x \cdot f(x, y, z)$

(b). $y \cdot(z x y z x+x z y x z)=y \cdot f(y, z, x)$ 
(c). $\quad z \cdot(x y z x y+y x z y x)=z \cdot f(z, x, y)$

where $f(x, y, z)=4\{(y \cdot z) x(y \cdot z)\}-\{y\{z x z\} y\}-\{z\{y x y\} z\}$

9. $2 y \cdot(x z y z x)=2 y \cdot\{x\{z y z\} x\}$

10(a). $\quad x^{2} \cdot\left(y^{2} z^{2}+z^{2} y^{2}\right)=2 x^{2} \cdot\left(y^{2} \cdot z^{2}\right)$

(b). $\quad y^{2} \cdot\left(z^{2} x^{2}+x^{2} z^{2}\right)=2 y^{2} \cdot\left(z^{2} \cdot x^{2}\right)$

(c). $z^{2} \cdot\left(x^{2} y^{2}+y^{2} x^{2}\right)=2 z^{2} \cdot\left(x^{2} \cdot y^{2}\right)$

11. $x^{2} \cdot(y z y z+z y z y)=2 x^{2} \cdot(y \cdot\{z y z\})=2 x^{2} \cdot(\{y z y\} \cdot z)$

12. $2 x^{2} \cdot\left(y z^{2} y\right)=2 x^{2} \cdot\left\{y z^{2} y\right\}$

13. $(x \cdot y) \cdot\left(x y z^{2}+z^{2} y x\right)=2(x \cdot y) R_{\left\{x y z^{2}\right\}}$

14. $(x \cdot y) \cdot(x z y z+z y z x)=2\{z y z\} R_{x} R_{x \cdot y}$

15. $(x \cdot y) \cdot\left(x z^{2} y+y z^{2} x\right)=2(x \cdot y) R_{\left\{x z^{2} y\right\}}$

16. $(x \cdot y) \cdot(z x y z+z y x z)=2(x \cdot y) \cdot\{z(x \cdot y) z\}=2\{(x \cdot y) z(x \cdot y)\} \cdot z$

T16 is clearly redundant, while use of formulae (3), (5) and (3) respectively shows that $\mathrm{T} 13, \mathrm{~T} 14$ and $\mathrm{T} 15$ are also redundant. So the set $T$ (namely T1-T12 together with those elements obtained from T1-T12 by permuting $x, y$ and $z$ ) spans $L$. We now display a set $U$ of Jordan elements. Each $U$-element may be considered as an element in $J_{0}^{(3)}$ : as such its expansion in $A^{(3)}$ appears as the corresponding $V$ element. Alternatively the $U$-element may be considered as an element in $L$ : its expression as a linear combination of $T$-elements appears as the corresponding $W$-element. For each integer $r$ the validity of the relation $U r=W r$ can be checked by appealing to MacDonald's theorem. For example, in the case of $r=7, U 7=W 7$ is valid in $J_{0}^{(3)}$ and linear

\begin{tabular}{|c|c|c|c|}
\hline $\mathrm{r}$ & $U$-elements & $V$-elements & $W$-elements \\
\hline 1 & $2 x^{2} \cdot\left\{y z^{2} y\right\}$ & $x^{2} y z^{2} y+y z^{2} y x^{2}$ & $\mathrm{~T} 12$ \\
\hline 2 & $2\left\{x\left\{y z^{2} y\right\} x\right\}$ & $2 x y z^{2} y x$ & $\mathrm{~T} 3-\mathrm{T} 12$ \\
\hline 3 & $2\left\{x^{2} y^{2} z^{2}\right\}$ & $x^{2} y^{2} z^{2}+z^{2} y^{2} x^{2}$ & $\mathrm{~T} 10 \mathrm{a}-\mathrm{T} 10 \mathrm{~b}+\mathrm{T} 10 \mathrm{c}$ \\
\hline 4 & $2\left\{x\left(y^{2} \cdot z^{2}\right) x\right\}$ & $x y^{2} z^{2} x+x z^{2} y^{2} x$ & $\mathrm{~T} 1-\mathrm{W} 3$ \\
\hline 5 & $2\{x(y \cdot\{z y z\}) x\}$ & $x y z y z x+x z y z y x$ & $\mathrm{~T} 2 \mathrm{a}+\mathrm{T} 2 \mathrm{~b}-\mathrm{T} 11$ \\
\hline 6 & $2\left\{x^{2}\{y z y\} z\right\}$ & $x^{2} y z y z+z y z y x^{2}$ & $\mathrm{~T} 2 \mathrm{a}-\mathrm{T} 2 \mathrm{~b}+\mathrm{T} 11$ \\
\hline 7 & $2\left\{z x^{2}\{y z y\}\right\}$ & $z x^{2} y z y+y z y x^{2} z$ & $\mathrm{~T} 4-\mathrm{W} 6$ \\
\hline 8 & $f\left(x^{2}, y, z\right)$ & $y z x^{2} y z+z y x^{2} z y$ & $\mathrm{~T} 6-\mathrm{W} 7$ \\
\hline 9 & $2 y \cdot\{x\{z y z\} x\}$ & $y x z y z x+x z y z x y$ & $\mathrm{~T} 9$ \\
\hline 10 & $2\{x y x\} \cdot\{z y z\}$ & $x y x z y z+z y z x y x$ & $\mathrm{~T} 5-\mathrm{W} 9$ \\
\hline 11 & $\begin{array}{l}x \cdot f(x, y, z)-y \cdot f(y, z, x) \\
\quad+z \cdot f(z, x, y)\end{array}$ & $x y z x y z+z y x z y x$ & $\mathrm{~T} 8 \mathrm{a}-\mathrm{T} 8 \mathrm{~b}+\mathrm{T} 8 \mathrm{c}$ \\
\hline
\end{tabular}


in $\{y z y\}$, so $U 7=W 7$ is valid in $J^{(3)}$. Suppose now that the sets of $U, V$ and $W$-elements have been augmented by adjoining all elements obtained from those displayed by permuting $x, y$ and $z$. The column headed $\pi$ shows the number of distinct elements obtained for each value of $r$. It is then easy to check that each $T$-element is a linear combination of $W$-elements, so the $W$-elements span $L$. But their images under $\nu$ are the $V$-elements which are clearly linearly independent. So $\nu \mid L$ is one-to-one. To complete the proof of the theorem we now prove the following lemma:

LEMMA 1. Let $n$ be an odd (positive) integer and $u$ an element in $K_{n} \cap L_{n}$ which is expressible in the form $u=\sum_{n=1}^{n} x_{i} \bullet y_{i}$. Then $y_{i} \in K_{n}$ for each $i=1, \cdots, n$.

Proof. For convenience we denote $\nu_{n}$ by $\nu_{\text {。 For }} n=1$ there is nothing to prove. Assume $n>1$ and let the coefficient of

$$
x_{i \div 1} x_{i+2} \cdots x_{n} x_{1} \cdots x_{i-1}\left(x_{2} \cdots x_{n} \text { if } i=1\right)
$$

in $y_{i} \nu$ be $\mu_{i}$ 。 Then the coefficient of $x_{\imath+1} x_{\imath+2} \cdots x_{n} x_{1} \cdots x_{i}$ in $2 u \nu$ is $\mu_{i}+\mu_{i+1}$. Since distinct monomials in $A^{(n)}$ are linearly independent we have $\mu_{i}+\mu_{i-1}=0, i=1, \cdots, n-1$ and $\mu_{n}+\mu_{1}=0$, whence $(n$ being odd) $\mu_{\imath}=0, i=1, \cdots, n$. In particular $\mu_{1}=0$, i.e. the coefficient of $x_{2} \cdots x_{n}$ in $y_{1} \nu$ is zero. It follows by considering suitable renumberings of $x_{2}, \cdots, x_{n}$ that $y_{1} \nu=0$, i.e. $y_{1} \in K_{n}$. Similarly $y_{i} \in K_{n}$ for $i=2, \cdots, n$.

Corollary. Let $u$ be an element in $K_{3}$ which is homogeneous of odd degree such that $u=x \cdot a+y \cdot b+z \circ c$. Then $a, b, c \in K_{3}$.

Proof. Suppose $u=x \cdot a+y \cdot b+z \cdot c$ is of degree $p$ in $x, q$ in $y$ and $r$ in $z$ with $p+q+r=n$ (an odd integer). Let $x_{1}, \cdots, x_{n}$ be $n$ symbols of which $p$ denote $x, q$ denote $y$ and $r$ denote $z$. For convenience we denote $\nu_{3}$ by $\nu$. For $n=1$ there is nothing to prove. We now proceed almost word for word as in the proof of the lemma. Assume $n>1$ and let $\mu_{i}$ be the coefficient of $x_{i+1} \cdots x_{n} x_{1} \cdots x_{i-1}\left(x_{2} \cdots x_{n}\right.$ if $i=1, x_{1} \cdots x_{n-1}$ if $\left.i=n\right)$ and so also of $x_{i-1} \cdots x_{1} x_{n} \cdots x_{i+1}\left(x_{n} \cdots x_{2}\right.$ if $i=1, x_{n-1} \cdots x_{1}$ if $i=n$ ) in the expansion in $A^{(3)}$ of $a \nu$ if $x_{i}=x$, of $b \nu$ if $x_{i}=y$ and of $c \nu$ if $x_{i}=z$. Then the coefficient of $x_{i+1} \cdots x_{n} x_{1} \cdots$ $x_{i}\left(x_{1} \cdots x_{n}\right.$ if $\left.i=n\right)$ in the expansion in $A^{(3)}$ of $2 u \nu$ is $\mu_{i}+\mu_{i+1}$ $\left(\mu_{n}+\mu_{1}\right.$ if $\left.i=n\right)$. Since distinct monomials in $A^{(3)}$ are linearly independent we have $\mu_{i}+\mu_{i+1}=0, i=1, \cdots, n-1$ and $\mu_{n}+\mu_{1}=0$. Whence ( $n$ being odd) $\mu_{i}=0, i=1, \cdots, n$. Since the argument goes through for any distribution of $p x$ 's, $q y$ 's and $r z$ 's amongst $x_{1}, \cdots, x_{n}$ the coefficient of each monomial in the expansion in $A^{(3)}$ of $a \nu$ is zero, i.e. $a \in K_{3}$. Similarly for $b$ and $c$. 
It is now sufficient for the proof of Theorem 2 to show that each element in $M$ is of the form $x \cdot a+y \cdot b+z \cdot c$. Let $N$ be the subspace of $J^{(3)}$ spanned by elements of this form. We shall write $a \equiv b$ to denote $a-b \in N$ : thus we wish to show that $m \equiv 0$ for each $m \in M$. Now $M$ is spanned by elements of the forms $a \cdot(b c d e f g+g f e d c b)$ and $(a \cdot b) \cdot(c d e f g+g f e d c)$ where two of $a, b, c, d, e, f, g$ represent $x$, two represent $y$ and three represent $z$. It is sufficient to show that each element of the form $(a \cdot b) \cdot(c d e f g+g f e d c)$ is in $N$, or by formulae (3), and (4) that each of the following is in $N$ :

$$
\begin{aligned}
& \text { (1) } a R_{b} R_{c} R_{d} R_{e} R_{f \cdot g}=c R_{a \cdot b} R_{d} R_{e} R_{f \cdot g} \\
& \text { (2) } a R_{b} R_{c} R_{d \cdot e} R_{f \cdot g}=c R_{a \cdot b} R_{d \cdot e} R_{f \cdot g} \\
& \text { (3) } a R_{b} R_{c \cdot d} R_{e} R_{f \cdot g}
\end{aligned}
$$

For types (1) and (2) let $t=a \cdot b \cdot c$. Then we have for $(1):(f \cdot g) R_{t \cdot d \cdot e}$, and for $(2):(f \cdot g) R_{d \cdot e \cdot t}$. Since $R_{t}=R_{a \cdot b \cdot c}$ it follows by two applications of formula (3) in each case that elements of types (1) and (2) are in $N$. Since any element in $M$ can be written as $z P$ where $P$ is an operator generated by the right multiplications $R_{u}, u \in J^{(3)}$, it will be sufficient in the case of elements of type (3) to consider $a=z$. The possibilities, modulo interchange of $x$ and $y$, are:

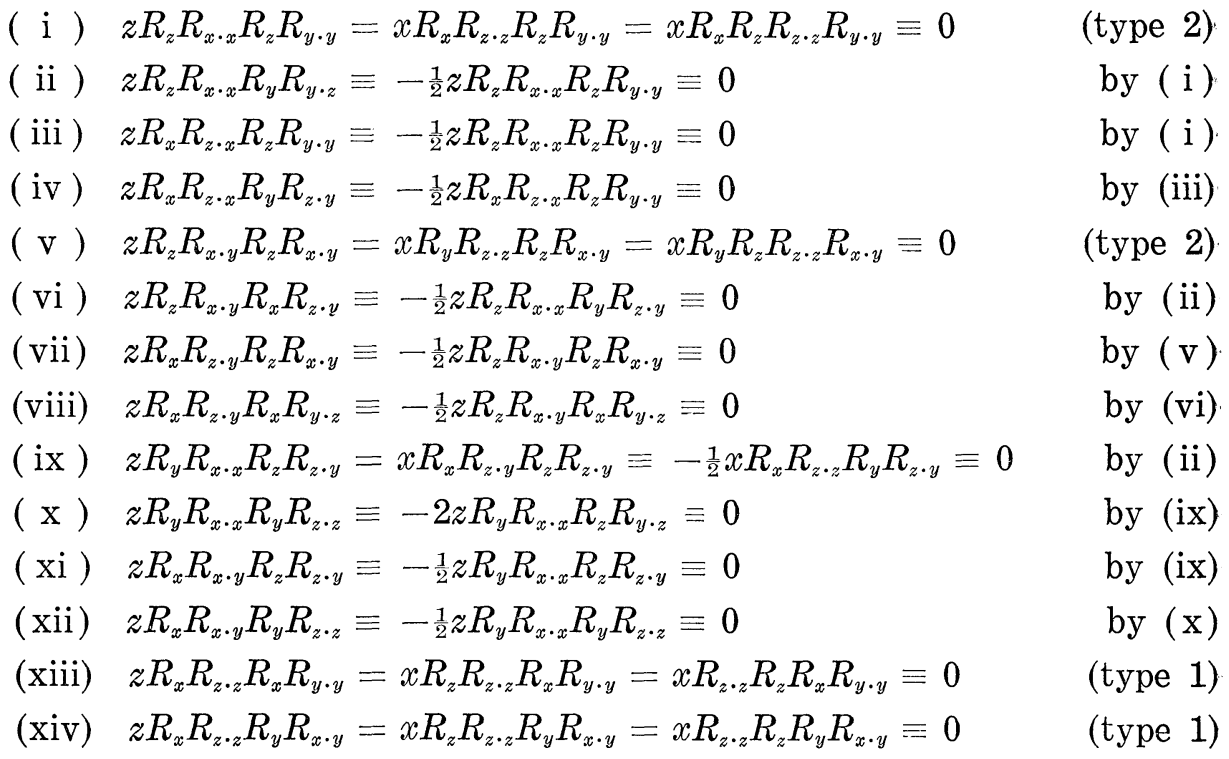

This completes the proof of Theorem 2 .

It is possible to avoid the use of MacDonald's theorem in the proof of Theorem 2 by using the following result, tabulating bases for each subspace spanned by homogeneous elements of degree six and applying 
the Corollary to Lemma 1 to each subspace spanned by homogeneous elements of degree seven. This process is straightforward, if somewhat tedious, and is in any case largely a special case of MacDonald's theorem. We include Theorem 3, however, as it would appear to be of independent interest, in providing easy verification of proposed five-variable identities linear in each variable.

\section{THEOREM 3. $K_{n} \cap L_{n}=\{0\}$ for $n \leqq 5$.}

Proof. The cases $n=1,2,3$ follow at once from the case $n=4$ with which we begin, taking the generators of $J^{(4)}$ as $x, y, z, t$. Let $R_{b}, S_{b c}, U_{b c}$ denote the mappings $a \rightarrow a \cdot b, a \rightarrow\{a b c\}, a \rightarrow\{b a c\}$ respectively. Then $S_{b c}=R_{b \cdot c}+R_{b} R_{c}-R_{c} R_{b}$, and $U_{b c}=R_{b} R_{c}+R_{c} R_{b}-R_{b \cdot c}$. Since $L_{4}$ is spanned by the elements $t R_{x} R_{y} R_{z}, t R_{x} R_{y \cdot z}, t R_{x \cdot y} R_{z}$ and all others obtained from these by permuting $x, y$ and $z$ and $R_{b \cdot c}=R_{b} R_{c}+R_{c} R_{b}-U_{b c}$, $2 R_{b} R_{c}=S_{b c}+U_{b c}$, we have that (again to within permutations of $x, y$ and $z) L_{4}$ is spanned by $t R_{x} S_{y z}, t R_{x} U_{y z}, t U_{x y} R_{z}$. Now let $u \in K_{4} \cap L_{4}$ and suppose that

$$
u=\Sigma t\left(\alpha_{x y z} R_{x} S_{y z}+\beta_{x} R_{x} U_{y z}+\gamma_{z} U_{x y} R_{z}\right)
$$

where the summation is over permutations of $x, y$ and $z$. Since $u \in K_{4}$ and distinct monomials in $A^{(4)}$ are linearly independent we have

(1) $\alpha_{x y z}=0$ (coefficient of txyz in $A^{(4)}$ ) and similarly each $\alpha$ coefficient is zero, and

(2) $\beta_{y}+\gamma_{z}=0$ (coefficient of $x t y z$ in $A^{(4)}$ ) and similarly for each pair of distinct subscripts. So $\beta_{x}=\beta_{y}=\beta_{z}=-\gamma_{x}=-\gamma_{y}=-\gamma_{z}$ and $u$ is a scalar multiple of

$$
t\left(R_{x} U_{y z}+R_{y} U_{z x}+R_{z} U_{x y}-U_{x y} R_{z}-U_{y z} R_{x}-U_{z x} R_{y}\right)
$$

which is zero by (5). So $K_{4} \cap L_{4}=\{0\}$.

The result for $n=5$ now follows by Lemma 1 and the fact, already noted in the proof of Theorem 2, that $L_{5}$ is spanned by the elements $a \cdot b$ where $a$ is a generator and $b$ is linear in each of the other generators.

2. In order to establish the existence of an $S$-identity of degree 8 we now examine the situation discussed by Albert and Paige in the paper [1] mentioned in the introduction.

Let $D$ be an algebra with an identity element 1 and an involution $d \rightarrow \bar{d}$. In the algebra $D_{n}$ of $n \times n$ matrices with entries in $D$ we can define an involution $M \rightarrow \bar{M}^{\prime}$ by taking $\left(\bar{M}^{\prime}\right)_{i j}=\left(\overline{M_{j i}}\right)$, i.e. $\bar{M}^{\prime}$ is the conjugate transpose of $M$. Further, we can define an involution $M \rightarrow M^{*}$ in $D_{n}$ by choosing a diagonal matrix $\Gamma=\operatorname{diag}\left\{\gamma_{1}, \cdots, \gamma_{n}\right\}$ 
where the $\gamma_{i}$ are self-adjoint $\left(\bar{\gamma}_{i}=\gamma_{i}\right)$, in the nucleus of $D$ and have inverses, and defining $M^{*}=\Gamma^{-1} \bar{M}^{\prime} \Gamma$. Such an involution is called a canonical involution in $D_{n}$. The particular case in which $\Gamma$ is the identity matrix reduces to the first involution defined and this is called a standard involution. It is clear that the subset of $D_{n}$ of matrices self-adjoint under a canonical involution (i.e. $M^{*}=M$ ) is closed under the product $A \cdot B=1 / 2(A B+B A)$ where $A B$ is the usual matrix product and forms an algebra relative to this product and the usual addition and scalar multiplication. We denote this algebra by $H\left(D_{n}, \Gamma\right)$ or simply $H\left(D_{n}\right)$ if $\Gamma$ is the identity matrix. With this notation the main theorem proved by Albert and Paige can be stated as:

Theorem 4. (Albert and Paige). If $H\left(D_{3}\right)$ is the homomorphic image of a special Jordan algebra then $D$ is associative.

Our first step will be to obtain a three-variable relation, $S(x, y, z)=$ 0 , which will be easily seen to hold in $J_{0}^{(3)}$ and so in any homomorphic image of a special Jordan algebra. Substitution of suitable elements $x, y, z$ from $H\left(D_{3}\right)$ will immediately show that $D$ is associative, giving an independent proof of the Albert-Paige result and simultaneously showing that $S(x, y, z)=0$ is not valid in every Jordan algebra, since an example is known (with $D$ as the eight-dimensional Cayley algebra) of a Jordan algebra $H\left(D_{3}\right)$ in which $D$ is not associative. The homogeneous part of $S(x, y, z)=0$ of degree 3 in $x, 2$ in $y$ and 3 in $z$ then gives the required $S$-identity of degree 8 . Lemmas 2 and 3 are essentially due to Albert and Paige.

LEMMA 2. Let $\theta$ be a homomorphism from a special Jordan algebra $H$, embedded in an associative algebra $U$, onto a Jordan algebra $J$ such that

(1) $H$ is generated by elements $X, Y, Z$ and $I$ ( $I$ an identity in $U)$ and

(2) $H$ contains elements $E_{1}, \cdots, E_{k}(k \geqq 3)$ such that $E_{i} E_{j}=$ $E_{j} E_{i}$ in $U$ and such that $e_{1}, \cdots, e_{k}\left(e_{i}=E_{i} \theta\right)$ form a set of orthogonal idempotents in $J$ whose sum is the identity $f=I \theta$ of $J$. Then, for $\alpha, \beta$ in the set $1, \cdots, k$ and $A$ a monomial in $U$ generated by $X, Y, Z$ and $I$ we have $\left(F_{\alpha} A F_{\beta}+F_{\beta} A^{*} F_{\alpha}\right) \theta \in J_{\alpha \beta}$ where $F_{\alpha}=E_{\alpha}^{2}, F_{\beta}=E_{\beta}^{2}, A^{*}$ is the reverse of $A$ and $J_{\alpha \beta}$ is the $\alpha, \beta$ component of $J$ in the Pierce decomposition determined by the $e_{i}$ 's.

Proof. Let $B=E_{\alpha} A E_{\beta}+E_{\beta} A^{*} E_{\alpha}, C=A+A^{*}$. Then

$$
\begin{aligned}
F_{\alpha} A F_{\beta}+F_{\beta} A^{*} F_{\alpha} & =E_{\alpha} B E_{\beta}+E_{\beta} B E_{\alpha}-\left(E_{\alpha} E_{\beta}\right) C\left(E_{\alpha} E_{\beta}\right) \\
& =2\left\{E_{\alpha} B E_{\beta}\right\}-\left\{\left(E_{\alpha} \cdot E_{\beta}\right) C\left(E_{\alpha} \cdot E_{\beta}\right)\right\}
\end{aligned}
$$


So

$$
\left(F_{\alpha} A F_{\beta}+F_{\beta} A^{*} F_{\alpha}\right) \theta=2\left\{e_{\alpha}(B \theta) e_{\beta}\right\}-\left\{\left(e_{\alpha} \cdot e_{\beta}\right)(C \theta)\left(e_{\alpha} \cdot e_{\beta}\right)\right\} \in J_{\alpha \beta}
$$

Lemma 2 '. With $H, J, \theta$ and condition (1) (but not condition (2)) as in Lemma 2 suppose that $E_{1}=1 / 2\left(X^{2}+X\right), E_{2}=I-X^{2}, E_{3}=$ $1 / 2\left(X^{2}-X\right)$ and $X \theta=x, I \theta=f, E_{1} \theta=e_{1}, E_{2} \theta=e_{2}, E_{3} \theta=e_{3}$. Then if (2) $x^{3}=x$, we have that (a) $e_{1}, e_{2}, e_{3}$ are orthogonal idempotents with sum $f$ and (b) $\left(E_{\alpha} A E_{\beta}+E_{\beta} A^{*} E_{\alpha}\right) \theta \in J_{\alpha \alpha}+J_{\alpha \beta}+J_{\beta \beta}$.

Proof. (a) This follows immediately from the definitions of $e_{1}$, $e_{2}, e_{3}$ and condition $(2)^{\prime}$.

(b) Let $B=X A(I-X)+(I-X) A^{*} X$. Then

$$
\begin{aligned}
2\left(E_{1} A E_{2}+E_{2} A^{*} E_{1}\right) \theta & =\{(I+X) B(I+X)\} \theta \\
& =\left\{\left(2 E_{1}+E_{2}\right) B\left(2 E_{1}+E_{2}\right)\right\} \theta \\
& =\left\{\left(2 e_{1}+e_{2}\right)(B \theta)\left(2 e_{1}+e_{2}\right)\right\} \in J_{11}+J_{12}+J_{22}
\end{aligned}
$$

Similarly for other choices of $\alpha$ and $\beta$.

Lemma 3. With notation as in Lemma 2':

$$
\begin{aligned}
& 2\left[\left(E_{\alpha} A E_{\beta}+E_{\beta} A^{*} E_{\alpha}\right) \cdot\left(E_{\beta} D E_{\gamma}+E_{\gamma} D^{*} E_{\beta}\right)\right] \theta \\
& \quad=\left[E_{\alpha} A E_{\beta} E_{\beta} D E_{\gamma}+E_{\gamma} D^{*} E_{\beta} E_{\beta} A^{*} E_{\alpha}\right] \theta
\end{aligned}
$$

where $D$ is a monomial in $U$ generated by $X, Y, Z$ and $I$, and $\alpha, \beta, \gamma$ are distinct integers chosen from 1, 2, 3.

Proof.

$$
\begin{aligned}
& 2\left[\left(E_{\alpha} A E_{\beta}+E_{\beta} A^{*} E_{\alpha}\right) \cdot\left(E_{\beta} D E_{\gamma}+E_{\gamma} D^{*} E_{\beta}\right)\right] \theta \\
&=\left(E_{\alpha} A E_{\beta} E_{\beta} D E_{\gamma}+E_{\gamma} D^{*} E_{\beta} E_{\beta} A^{*} E_{\alpha}\right) \theta \\
&+\left(E_{\alpha} A E_{\beta} E_{\gamma} D^{*} E_{\beta}+E_{\beta} D E_{\gamma} E_{\beta} A^{*} E_{\alpha}\right) \theta \\
&+\left(E_{\beta} A^{*} E_{\alpha} E_{\beta} D E_{\gamma}+E_{\gamma} D^{*} E_{\beta} E_{\alpha} A E_{\beta}\right) \theta \\
&+\left(E_{\beta} A^{*} E_{\alpha} E_{\gamma} D^{*} E_{\beta}+E_{\beta} D E_{\gamma} E_{\alpha} A E_{\beta}\right) \theta
\end{aligned}
$$

Now, since $\alpha, \beta$ and $\gamma$ are distinct, $J_{\alpha \beta} J_{\beta \gamma} \subseteq J_{\alpha \gamma \bullet}$. So, by Lemma $2^{\prime}$, the left-hand-side is in $J_{\alpha \gamma}$. The result now follows from Lemma $2^{\prime}$ and the disjointness of the Peirce decomposition.

Corollary.

$$
\begin{aligned}
& 4\left[\left\{E_{1} Z E_{2}\right\} \cdot\left(E_{2} A E_{2} E_{2} Z E_{3}+E_{3} Z E_{2} E_{2} A^{*} E_{2}\right)\right] \theta \\
& \quad=4\left[\left(E_{1} Z E_{2} E_{2} A E_{2}+E_{2} A^{*} E_{2} E_{2} Z E_{1}\right) \cdot\left\{E_{2} Z E_{3}\right\}\right] \theta
\end{aligned}
$$

Equation (6) suggests the following relation in $U$ : 


$$
\begin{aligned}
4\left[\left\{E_{1} Z E_{2}\right\} \cdot\left(E_{2} C E_{2} E_{2} Z E_{3}+E_{3} Z E_{2} E_{2} C^{*} E_{2}\right)\right] \\
\quad-\left(E_{2} Z E_{1} E_{2} C E_{2} E_{2} Z E_{3}+E_{3} Z E_{2} E_{2} C^{*} E_{2} E_{1} Z E_{2}\right) \\
\quad-\left(E_{1} Z E_{2} E_{3} Z E_{2} E_{2} C^{*} E_{2}+E_{2} C E_{2} E_{2} Z E_{3} E_{2} Z E_{1}\right) \\
\quad-\left(E_{2} Z E_{1} E_{3} Z E_{2} E_{2} C^{*} E_{2}+E_{2} C E_{2} E_{2} Z E_{3} E_{1} Z E_{2}\right) \\
=4\left[\left(E_{1} Z E_{2} E_{2} C E_{2}+E_{2} C^{*} E_{2} E_{2} Z E_{1}\right) \cdot\left\{E_{2} Z E_{3}\right\}\right] \\
\quad-\left(E_{1} Z E_{2} E_{2} C E_{2} E_{3} Z E_{2}+E_{2} Z E_{3} E_{2} C^{*} E_{2} E_{2} Z E_{1}\right) \\
\quad-\left(E_{2} C^{*} E_{2} E_{2} Z E_{1} E_{2} Z E_{3}+E_{3} Z E_{2} E_{1} Z E_{2} E_{2} C E_{2}\right) \\
\quad-\left(E_{2} C^{*} E_{2} E_{2} Z E_{1} E_{3} Z E_{2}+E_{2} Z E_{3} E_{1} Z E_{2} E_{2} C E_{2}\right)
\end{aligned}
$$

where, for reasons which will appear later, we take $C=Y X Z Y$ and $C^{*}=Y Z X Y$. In turn, (7) suggests the following relation in $J_{0}^{(3)}$, (this is the relation referred to previously as $S(x, y, z)=0$ )

$$
\begin{aligned}
4\left\{e_{1} z e_{2}\right\} & \cdot p_{1}+\left\{\left(e_{2}+2 e_{3}\right) q_{1}\left(e_{2}+2 e_{3}\right)\right\} \\
& -\left\{\left(2 e_{1}+e_{2}\right) r_{1}\left(2 e_{1}+e_{2}\right)\right\}-\left\{e_{2} s_{1} e_{2}\right\} \\
= & 4\left\{e_{2} z e_{3}\right\} \cdot p_{2}-\left\{\left(2 e_{1}+e_{2}\right) q_{2}\left(2 e_{1}+e_{2}\right)\right\} \\
& +\left\{\left(e_{2}+2 e_{3}\right) r_{2}\left(e_{2}+2 e_{3}\right)\right\}-\left\{e_{2} s_{2} e_{2}\right\}
\end{aligned}
$$

where $e_{1}=1 / 2\left(x^{2}+x\right), e_{2}=1-x^{2}, e_{3}=1 / 2\left(x^{2}-x\right)$ and $p_{1}, 2 q_{1}, 2 r_{1}, s_{1}, p_{2}$, $2 q_{2}, 2 r_{2}, s_{2}$ are Jordan elements in $J_{0}^{(3)}$ equal respectively in $A^{(3)}$ to

$$
\begin{aligned}
& e_{2} y x z y e_{2} e_{2} z e_{3}+e_{3} z e_{2} e_{2} y z x y e_{2}, \\
& (1+x) z e_{1} e_{2} y x z y e_{2} e_{2} z x+x z e_{2} e_{2} y z x y e_{2} e_{1} z(1+x), \\
& x z e_{2} e_{3} z e_{2} e_{2} y z x y(1-x)+(1-x) y x z y e_{2} e_{2} z e_{3} e_{2} z x, \\
& z e_{1} e_{3} z e_{2} e_{2} y z x y+y x z y e_{2} e_{2} z e_{3} e_{1} z, \\
& e_{1} z e_{2} e_{2} y x z y e_{2}+e_{2} y z x y e_{2} e_{2} z e_{1}, \\
& x z e_{2} e_{2} y x z y e_{2} e_{3} z(1-x)+(1-x) z e_{3} e_{2} y z x y e_{2} e_{2} z x, \\
& (1+x) y z x y e_{2} e_{2} z e_{1} e_{2} z x+x z e_{2} e_{1} z e_{2} e_{2} y x z y(1+x), \\
& y z x y e_{2} e_{2} z e_{1} e_{3} z+z e_{3} e_{1} z e_{2} e_{2} y x z y .
\end{aligned}
$$

Now, (8) is an S-identity. By construction it holds in $J_{0}^{(3)}$ and we may see that it does not hold in $H\left(C_{3}\right)$, where $C$ is the eight-dimensional Cayley algebra, by substituting

$$
x=\left(\begin{array}{rrr}
1 & \cdot & \cdot \\
\cdot & \cdot & \cdot \\
\cdot & \cdot & -1
\end{array}\right), \quad y=\left(\begin{array}{ccc}
\cdot & 1 & \cdot \\
1 & \cdot & 1 \\
\cdot & 1 & \cdot
\end{array}\right), \quad z=\left(\begin{array}{ccc}
\bullet & u & v \\
\bar{u} & \bullet & w \\
\bar{v} & \bar{w} & \cdot
\end{array}\right)
$$

where $u, v$ and $w$ are arbitrary elements in $C$, and examining the 1,3 element on each side of (8). The calculation is quite simple: by choice of $x$, the only nonzero contribution on each side arises from the first term. Further, $p_{1}$ and $p_{2}$ are of degree two in $z$ and so may be evaluated as though $C$ were associative, that is by substituting directly 
into their equivalent associative forms displayed above. The result is $u[(v-\bar{v}) w]$ on the left and $[u(v-\bar{v})] w$ on the right. Since self-adjoint elements in $C$ are in any case in the nucleus we have $u[(v+\bar{v}) w]=$ $[u(v+\bar{v})] w$ Whence $u(v w)=(u v) w$. But $C$ is not associative. So (8) does not hold in the Jordan algebra $H\left(C_{3}\right)$ and is thus an $S$-identity.

The relation (8) can be written as $\sum_{i=3}^{15} f_{i}(x, y, z)=0$, where $f_{i}(x, y, z)$ is a Jordan polynomial of degree $i$ in $x$. Now $f_{i}(x, y, z)$ can be expanded in $A^{(3)}$ as a linear combination of monomials in $x, y, z$ of degree $i$ in $x$. Since $A^{(3)}$ is free, $f_{i}(x, y, z)=0$ for each $i$. We consider the case $i=3$.

The parts of the terms of (8) which are of degree 3 in $x$ are equal respectively in $A^{(3)}$ to:

$$
\begin{aligned}
& -4(x \cdot z) \cdot(y x z y z x+x z y z x y) \\
& z x y x z y z x+x z y z x y x z \\
& x z x z y z x y+y x z y z x z x \\
& z x x z y z x y+y x z y z x x z \\
& -4(z \cdot x) \cdot(x z y x z y+y z x y z x) \\
& x z y x z y x z+z x y z x y z x \\
& y z x y z x z x+x z x z y x z y \\
& y z x y z x x z+z x x z y x z y
\end{aligned}
$$

We now make the following choices for Jordan expressions of the above:

$$
\begin{gathered}
\text { (a) }+(\mathrm{c})+(\mathrm{d}):-4\{(x \cdot z) y\{x\{z y z\} x\}\} \\
(\mathrm{b}): 4\{\{z\{x y x\} z\} y(z \cdot x)\}-2\{z\{x\{y(x \cdot z) y\} x\} z\} \\
(\mathrm{e})+(\mathrm{f})+(\mathrm{g})+(\mathrm{h}):-2\{x\{z\{y(x \cdot z) y\} z\} x\}
\end{gathered}
$$

and obtain the following relation which clearly holds identically in $J_{0}^{(3)}$ :

$$
\begin{aligned}
& 4\{\{z\{x y x\} z\} y(z \cdot x)\}-2\{z\{x\{y(x \circ z) y\} x\} z\} \\
& \quad=4\{(x \cdot z) y\{x\{z y z\} x\}\}-2\{x\{z\{y(x \cdot z) y\} z\} x\}
\end{aligned}
$$

Substitution in (9) of the same elements as were substituted in (8) shows that (9) is an $S$-identity.

3. In $H\left(D_{3}\right)$ let

$$
g=\left(\begin{array}{lll}
\alpha & p & q \\
\bar{p} & \beta & r \\
\bar{q} & \bar{r} & \gamma
\end{array}\right), \quad x=\left(\begin{array}{ccc}
\cdot & 1 & \cdot \\
1 & \cdot & \cdot \\
\cdot & \cdot & \cdot
\end{array}\right), \quad y=\left(\begin{array}{lll}
\cdot & \cdot & \cdot \\
\cdot & \cdot & 1 \\
\cdot & 1 & \cdot
\end{array}\right) .
$$

Then we have 


$$
\{x g x\}=\left(\begin{array}{ccc}
\beta & \bar{p} & \cdot \\
p & \alpha & \cdot \\
\cdot & \cdot & \cdot
\end{array}\right) \text { and }\{y g y\}=\left(\begin{array}{ccc}
\cdot & \bullet & \bullet \\
\cdot & \gamma & \bar{r} \\
\cdot & \gamma & \beta
\end{array}\right) \text {, }
$$

while $x g y$ (ordinary matrix multiplication) is equal to

$$
\left(\begin{array}{lll}
\cdot & r & \beta \\
\cdot & q & p \\
\cdot & \cdot & \cdot
\end{array}\right)
$$

With these results in mind, (8) suggests the following candidate for an $S$-identity:

$$
\begin{aligned}
2\{x z x\} & \cdot\left\{x\left\{z y^{2} z\right\} y\right\}-\{x\{z\{x\{y z y\} y\} z\} x\} \\
= & 2\left\{x\left\{z x^{2} z\right\} y\right\} \cdot\{y z y\}-\{y\{z\{y\{x z x\} x\} z\} y\}
\end{aligned}
$$

We verify that (10) is an S-identity by using it to prove the AlbertPaige Theorem in a slightly strengthened form. (Albert and Paige mention that their method will give the stronger result but do not give the details.)

Theorem 4(a). If $H\left(D_{n}, \Gamma\right), n \geqq 3$, is the homomorphic image of a special Jordan algebra then $D$ is associative.

[Theorem 4(a) is also a stronger form of a theorem due to Jacobson [3] viz: If $H\left(D_{n}, \Gamma\right), n \geqq 3$, is a special Jordan algebra then $D$ is associative.]

Proof of Theorem 4(a). It is sufficient to prove the result for $n=3$. Since $H\left(D_{3}, \Gamma\right)$ is the homomorphic image of a special Jordan algebra the relation (10), which clearly holds in $J_{0}^{(3)}$, holds in $H\left(D_{3}, \Gamma\right)$. Now suppose that

$$
\Gamma=\left(\begin{array}{ccc}
\alpha & \bullet & \bullet \\
\cdot & \beta & \bullet \\
\cdot & \bullet & \gamma
\end{array}\right)
$$

and let

$$
x=\left(\begin{array}{ccc}
\cdot & \beta & \cdot \\
\alpha & \cdot & \cdot \\
\cdot & \cdot & \cdot
\end{array}\right), \quad y=\left(\begin{array}{ccc}
\cdot & \cdot & \cdot \\
\cdot & \cdot & \gamma \\
\cdot & \beta & \cdot
\end{array}\right), \quad z=\left(\begin{array}{lll}
\cdot & \bar{u} \beta & v \gamma \\
u \alpha & \cdot & \bar{w} \gamma \\
\bar{v} \alpha & w \alpha & \cdot
\end{array}\right),
$$

where $u, v$ and $w$ are arbitrary elements in $D$. Substitution in (10) gives, in the first row, third column:

left hand side: $\beta w \alpha \beta(\alpha v \gamma \beta \gamma w \beta \gamma)$

right hand side: $(\beta u \alpha \beta \alpha v \gamma \beta) \gamma w \beta \gamma$ 
Since $u, v$ and $w$ are arbitrary and $\alpha, \beta$ and $\gamma$ are in the nucleus of $D$ with inverses the result follows at once.

REMARK. It can be shown by using the corollary to Lemma 1 that the $S$-identity (10) is generated by $S$-identities of degree 8 . We do not give the details here as we hope to embody them in a later paper.

\section{REFERENCES}

1. A. A. Albert and L. J. Paige, On a homomorphism property of certain Jordan algebras, Trans. Amer. Math. Soc. 93 (1959), 20-29.

2. P. M. Cohn, On homomorphic images of special Jordan algebras, Canadian J. of Math. 6 (1954), 253-264.

3. N. Jacobson, Structure of alternative and Jordan bi-modules, Osaka Math. J. 6 (1954), 1-71.

4. I. G. MacDonald, Jordan algebras with three generators, Proc. London Math. Soc. series 3, 10 (1960), 395-408. 



\section{PACIFIC JOURNAL OF MATHEMATICS}

EDITORS

H. SAMELSON

Stanford University

Stanford, California

R. M. BLUMENTHAL

University of Washington

Seattle, Washington 98105
*J. DUGUNDJI

University of Southern California

Los Angeles, California 90007

RICHARD ARENS

University of California

Los Angeles, California 90024

\section{ASSOCIATE EDITORS}

E. F. BECKENBACH

B. H. NeumanN

F. WOLF

K. YosidA

\section{SUPPORTING INSTITUTIONS}

UNIVERSITY OF BRITISH COLUMBIA

CALIFORNIA INSTITUTE OF TECHNOLOGY

UNIVERSITY OF CALIFORNIA

MONTANA STATE UNIVERSITY

UNIVERSITY OF NEVADA

NEW MEXICO STATE UNIVERSITY

OREGON STATE UNIVERSITY

UNIVERSITY OF OREGON

OSAKA UNIVERSITY

UNIVERSITY OF SOUTHERN CALIFORNIA
STANFORD UNIVERSITY

UNIVERSITY OF TOKYO

UNIVERSITY OF UTAH

WASHINGTON STATE UNIVERSITY

UNIVERSITY OF WASHINGTON

AMERICAN MATHEMATICAL SOCIETY CHEVRON RESEARCH CORPORATION

TRW SYSTEMS

NAVAL ORDNANCE TEST STATION 


\section{Pacific Journal of Mathematics}

\section{Vol. 16, No. $1 \quad$ November, 1966}

Larry Armijo, Minimization of functions having Lipschitz continuous first

partial derivatives ............................... 1

Edward Martin Bolger and William Leonard Harkness, Some

characterizations of exponential-type distributions.............. 5

James Russell Brown, Approximation theorems for Markov operators ...... 13

Doyle Otis Cutler, Quasi-isomorphism for infinite Abelian p-groups ...... 25

Charles M. Glennie, Some identities valid in special Jordan algebras but not valid in all Jordan algebras .......................... 47

Thomas William Hungerford, A description of $\operatorname{Mult}_{i}\left(A^{1}, \cdots, A^{n}\right)$ by

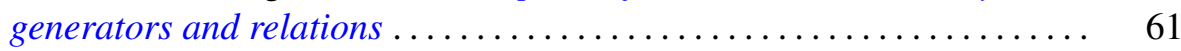

James Henry Jordan, The distribution of cubic and quintic non-residues ....

Junius Colby Kegley, Convexity with respect to Euler-Lagrange differential operators................................... 87

Tilla Weinstein, On the determination of conformal imbedding ......... 113

Paul Jacob Koosis, On the spectral analysis of bounded functions ........ 121

Jean-Pierre Kahane, On the construction of certain bounded continuous functions ................................... 129

V. V. Menon, A theorem on partitions of mass-distribution ........... 133

Ronald C. Mullin, The enumeration of Hamiltonian polygons in triangular

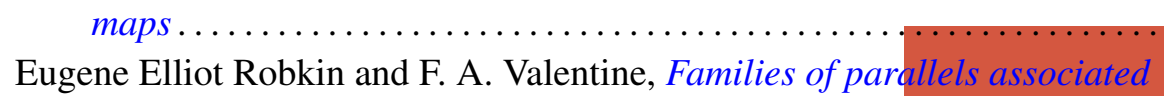

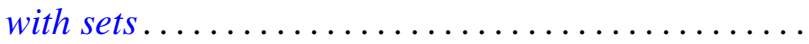

Melvin Rosenfeld, Commutative F-algebras

A. Seidenberg, Derivations and integral closure

S. Verblunsky, On the stability of the set of exponents of a Cauchy

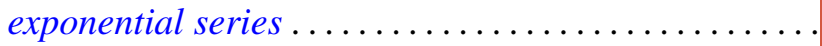

Herbert Walum, Some averages of character sums 